\title{
Approach to Financial Decision Making within Quantum Physics and Neurosciences
}

\author{
Ana Njegovanović \\ Correspondence: Ana Njegovanović, Zagreb, Kamaufova 3, Croatia.
}

Received: May 6, 2020

Accepted: May 18, 2020

Online Published: September 2, 2020

doi:10.11114/ijlpa.v3i2.4991

URL: https://doi.org/10.11114/ijlpa.v3i2.4991

\begin{abstract}
This paper focuses on the study of the functional relationships between the tools of neuroscience, neurofinance and psychology on the one hand, and quantum physics / quantum mechanics and neurophysiology on the other. Can physics / quantum mechanics help explain / understand human behavior through the Shrödinger cat platform (perhaps we can explain the most mysterious phenomena: human behavior - cerebral secretion?)? The concepts of quantum mechanics allow a good prediction of human decision making within Schrödinger's cat (two particles can talk to each other even at a distance of a galaxy, perhaps in this sense can help explain an extremely complex decision making system), and define the "connection of quantum models with neurophysiological processes in the brain "... which is a very complex problem." (Haven and Khrennikov) The application of quantum physics and neuroscience in finance allows us to consider the complexity of financial decision making, while the connection between quantum physics and psychology manifests itself as the field of quantum physics seeks to understand the fundamental nature of particles. while the field of psychology seeks to explain human nature along with its inherent misconceptions.If decision-making is a process of gathering evidence in favor of different alternatives over time, the process is discontinued once the decision limit is reached, followed by choice of decision. e activity within the posterior parietal cortex several important questions remain unanswered. Neural mechanisms that support the accumulation of evidence record the activities of individual neurons in different parts of the prefrontal cortex (PFC) and the lateral intraparietal area (LIP).
\end{abstract}

Keywords: neuroscience, psychology, financial decision making, quantum physics, quantum mechanics, neurophysiology

\section{Introduction}

This paper focuses on the study of the functional relationships between the tools of neuroscience, neurofinance and psychology on the one hand, and quantum physics / quantum mechanics and neurophysiology on the other. Can physics / quantum mechanics help explain / understand human behavior through the Shrödinger cat platform (perhaps we can explain the most mysterious phenomena: human behavior - cerebral secretion?)? The concepts of quantum mechanics allow a good prediction of human decision making within Schrödinger's cat (two particles can talk to each other even at a distance of a galaxy, perhaps in this sense can help explain an extremely complex decision making system), and define the "connection of quantum models with neurophysiological processes in the brain "... which is a very complex problem." (Haven and Khrennikov) The application of quantum physics and neuroscience in finance allows us to consider the complexity of financial decision making, while the connection between quantum physics and psychology manifests itself as the field of quantum physics seeks to understand the fundamental nature of particles. while the field of psychology seeks to explain human nature along with its inherent misconceptions. Both scientific disciplines seek to predict how systems will behave in the future. Unlike classical decision theory, the quantum world leaves room for a certain degree of uncertainty.

Namely, the terms uncertainty - the central principle of quantum theory states great uncertainty in the world of very small, while the big world can predict (for example: when a train is on the road, speed data can predict the arrival of a train at the next station, while electrons can not accurately know the place and moment of the electron but we can calculate the probability that a particle can appear at a particular place).

The interaction of the tools of physics and finance enables us to understand new knowledge in dealing with the various risks found in finance. Concepts in physics can explain financial market phenomena that are complex in structures and thus confuse investors. Modern science and technology, including electronics, nuclear technology, nanotechnology, 
femto-chemistry, molecular biology, cosmology, high-energy physics, quantum mechanics, are essential in understanding financial markets. We can identify money and the brain are quantum phenomena, financial markets and financial decision making are the intersection of neuroscience, neurofinance and quantum physics.

Investors' investment decisions are influenced by a variety of biological factors that provide us with information and explanations for investor behavior using neuroscience tools to understand how the brain analyzes election information in the face of monetary uncertainties and the neural processes involved in decision making. The interaction of neuroscience and financial decision-making has opened up an interdisciplinary field of neurofinance, a discipline that provides an understanding of the significant neurophysiological foundations underlying various cognitive processes and behaviors (electrophysiology, psychology and psychophysiology contribute to the field of neurofinance development).

From the aspect of quantum mechanics, the outcome of the decision made (good or bad decision) reflects their final choice. If the decision-making process of the human mind can follow the quantum behavior of mechanics, then we can expect applicability in other fields that have directly influenced decision-making. Research conducted in the application of finance and quantum mechanics (Kondratenko 2005; Baaquie 2004; Baaquie 2009a) and the application of quantum mechanics in economics / finance were evident in price dynamics models (Choustova 2007), stock prices (Schaden 2003; Bagarello 2009), interest rates (Baaquie 2009b), inclusion of private data (Ishio and Haven 2009; Haven 2008).

We could explain the modeling of probable and irrational behavioral phenomena by quantum theory, which was initially developed to describe probable processes in the physics of atom size (Orlov, 1981; Peres, 2002; Aerts, 2009; Busemeyer and Wang, 2015; Khrennikov, 2015). The approach is based on the self-consistent methodological background of quantum theory, and benefits from its embedded contextual probabilistic nature. It enables the production of quantitative models for various behavioral phenomena that compromise the classical paradigm of rational-agent issues: sequence effects, risk of ambiguity aversion, altruistic collaboration, social and market instability, connectedness, disjunction, and other "misconceptions" of human logic (Khrennikov, 2010; Busemeyer and Bruza , 2012; Haven and Khrennikov, 2013, 2017; Aerts et al., 2016b). This quantum approach to human cognition and behavior supports the assumption that the mathematical formalism of quantum theory is a basic computation of contextually probable phenomena in nature (Gabora and Aerts, 2005).

As Lynch (1995) points out, a feature of quantum theory in which phase does not have a good definition of observation. Namely, if we observe human thinking in transitional amplitudes, it allows us to expand cognitive alternatives, we deviate from the classical set of theoretical probability calculations (corresponding to the rational logic of logic). If we start from the fact that quantum decision making is a transitional subject from an indefinite to a certain cognitive state in relation to collective alternative choices, then they are mutually exclusive forming an orthogonal base in a complex vector space where the cognitive state $\Phi$ is represented as a vector (or sub-space of larger dimension). $\mid \Psi>$. In this state, the probability of making decision A corresponds to the corresponding cognitive state $\mathrm{A}\rangle$ is given by the quadratic modulus of complex overlap values $\langle\Psi \mid A\rangle$, known in quantum theory as the amplitude of the transition between the corresponding states. In doing so, transition amplitudes characterize amplitude and phase, with the nature of the quantum being the determinant of the quantum approach to cognition. In contrast to amplitude, the transition phase is not determined directly by the measurable probability of the corresponding decision.

The Schrödinger equation can be transformed into an equation of options using variable transformations, and then two types of potentials are considered, one potential without arbitration and two with arbitration. Haven (2002) showed that such potential functions are crucial for option pricing theory. and arbitrage prices are obtained only when the function is converted into one. Solutions of the modified equation can be obtained by the WKB approximation method or by numerical simulation methods.

Thus, the application of the Schrödinger equation principle in Black-Scholes price options like many other scientists, such as, Haven $(2002,2003)$, Khrennikov and Haven, (2013), tried to construct an option price through an alternative BlackScholes pricing model. as a state function to find a potential function for solving the eigenvalues of an equation. Haven 's (2003) extension where the uncertainty in the original option price is replaced by quantum information set by Qubit whose superposition state is 0 and 1 .

\section{Literature Review}

Quantum modeling in its beginnings has been successful in terms of choosing ambiguous data or data on asset movements (Khrennikov and Haven, 2013). The philosophical aspect of classical decision theory (and other philosophers) was based on Boolean philosophy of determinism and logic (Kolgomorova 1933; Khrennikov and Haven, 2013, 2009; Savage, 1954; Knight, 1921).

Consideration of probabilities in classical decision theory (as well as in classical physics, including the theory of relativity) and has an episemic nature (Bayesian theory). The inconsistency of solving the problem of measuring quantum physics 
as two "incompatible processes" in theory (Roger Penrose, 1989), the unique evolutionary time is represented by the Schrödinger equation (process $\mathrm{U}$ ) and the collapse of the state vector / wave function during measurement (R process). Applying this model to a decision-making (behavior) model does not build a physical theory of measurement. The classical theory of measurement that was expected (von Helmholtz) and elaborated (Campbell) contains a formulation called the law of measurement.

Namely, measurement disturbs Schrödinger's equation due to a peculiar phenomenon called quantum feedback. Quantum measurement affects the system being observed: The act of observing introduces some kind of random noise into the system. This is ultimately the source of Heisenberg's famous principle of uncertainty. The uncertainty of the measurement is not, as Heisenberg says, the result of a clumsy intervention in a sensitive quantum system - a photon that hits a particle and pushes it out of direction. Instead, it is the inevitable outcome of a truly randomized effect of the observation itself. Schrödinger's equation predicts quite well how a quantum system develops - unless you measure it, in which case the result is unpredictable.

The neoclassical theory of financial economics (basic assumptions) is based on axiomatic formulations (general equilibrium theory / market hypothesis, social choice theory / standard game theory). By choosing an agent in a competitive market, their maximization of a certain useful function is noticeable, as well as the existence of a mathematical assumption that there is no asymmetry and uncertainty of information. These assumptions lead us to Paret's optimal result. (Arrow - Debreu model of general equilibrium as an epithet of such axiomatic modeling based on setteoretical real analysis).

Keynes (1920) and others pointed out that there is uncertainty in economics that is difficult to describe by standard probability models. Accordingly, Boolean standard logical operations do not describe the behavior of agents. Cognitive scientists (Kahneman, Tversky, Shaffir) and other scientists have explored financial behavior that has resulted in good models, perspective theory (Kahnemana, 1992), heuristic theory, and Bayesian learning models, with limitations; thus, the "zero previous trap" of Bayesian modeling (Bajs et al., 2017) means that no significant posterior probability update can be obtained if previously 0 or 1 . However, in financial markets, large belief updates are not uncommon and are most pronounced in crisis times.

In finance, there is a visible difference in the perception / knowledge / opinion (Miller, 1977) of investors in stock markets, which cannot be reduced to the problem of information asymmetry. Asymmetric information theory states that an imbalance of information between buyers and sellers can lead to market failure. Market failure (to economists) means the inefficient distribution of goods and services in the free market, in which prices are determined by the law of supply and demand. However, in many cases price bubbles are formed in a scenario of uncertainty or divergence of opinion between agents, this cannot be easily explained by models of information asymmetry. So, a different modeling model is needed.

Khrennikov Polina and Patra Sudip (2019) developed a model of quantum agent belief based on uncertainty in financial markets. They opened up an approach to an alternative formulation of the overall law of probability, the basis for describing the movement of property prices in uncertainties. Decision-making is a complex and intertwined network, some clarifications can provide us with a better understanding of the decision-making process in conditions of uncertainty: (A Quantum framework for economic science: new directions, Discussion Paper, 2019; interpretation from 1 to 7)

1. Experimental data on actual decision-making present a challenge to the expected utility theory, prediction (Kahneman).

2. Ellsberg (1961) pointed out violations of the principle of expected utility theory (EUT) in uncertain situations, it is necessary to emphasize that situations are different from risky situations.

3. Violation of the rules of Kolmogor's law of probability: 'Errors in decision-making. People prefer action A over action $\mathrm{B}$ if they know that event $\mathrm{E}$ happened and if they know that $\mathrm{E}$ does not happen, but they prefer B over $\mathrm{A}$ if they do not know if $\mathrm{E}$ is happening or not.

4. Effects of order $\mathrm{P}(\mathrm{A} \& \mathrm{~B})$ ne $=\mathrm{P}(\mathrm{B} \& \mathrm{~A})$, where $\mathrm{P}$ means probability and $\mathrm{A}$ and $\mathrm{B}$ are mutually exclusive events. This equality is evident in classical decision theory, but based on Hilbert's cosmic formulation of quantum decision theory, such equality can be violated, as evidenced by experimental data on actual behavior.

5. Violation of the law of monotony of Kolmogor's probability: "Probability errors". People rate the conjunction event 'A and B' (disjunction event 'A or B') as more (less) likely that events A and / and B are separate.

6. Failure of the law of total probability: Data on behavior in conditions of uncertainty violates the law of total probability (Khrennikov and Haven, 2009, 2013)

7. Quantum modeling can potentially help address current anomalies, in explaining a decision with uncertainty (Gilboa, Postlewaite, Schmeidler, 2008).

\section{Neuroscience, Neurofinance, Psychology and Financial Decision}


Decision-making research relies on psychology, neurobiology, pathology as well as economics and covers factors that play a major role in the neural-level decision-making process, whether consciously or subconsciously. From a psychological standpoint, decision making is a process in which the cognitive, emotional and motivational aspects play a vital role. Imaging brain fMRIs reveal that decision-making processes begin before the individual is capable of realization. Neurochemistry has identified several neurotransmitters that are differentially linked to decision-making processes, the most important of which are dopamine, serotonin, cortisol, oxytocin and prolactin. The complex nature of neurotransmitters, the mechanisms that implicate their production, must be fully understood and it is not yet fully known how they work. From a neurofinance / neuroeconomics perspective, control of the decision-making process is determined by good communication among different parts of the brain that is regulated by serotonin levels. Decision-making is a complex process that is possible because of the processes that take place in many parts of our brain. Neuroanatomically, the prefrontal cortex plays a key role in coordinating these processes. To some extent, decision making is based on the assumption that people are able to predict other people's behavior. This ability arises from individual preferences and beliefs. Social neuroscience allows us to see the neural mechanisms underlying human ability to represent our intentions. Neurobiology, in turn, seeks to explain how relevant moral decisions occur in our brains and how they can modify our emotions. The neurobiological background of our decision-making processes give us insight into the supposed limited human rationality as well as the role of emotions, morals and empathy. At the same time, neuroscientific research contributes to our knowledge of the flow of decision-making processes and their adaptive value.

Different brain regions work together during the decision-making process.

A February 2015 study from the University of Okinawa Institute of Science and Technology (OIST) in Japan found that a key part of the brain involved in decision-making, called the striatum, functions hierarchically within its three different sub-regions.

The striatum is part of the basal ganglia, which form the inner core of the brain and process both decision making and subsequent actions. Neuroscientists divide the striatum into three regions: 1. Ventral (VS), 2. Dorsomedial (DMS), and 3. Dorsolateral (DLS). Each region has a characteristic role in: 1. Motivation 2. Adaptable decisions and 3. Routine actions.

Research findings indicate that "Different neural representations in the dorsolateral, dorsomedial, and ventral part of the striate during fixed and free choice tasks" (Neuroscience, 2015). Although the three different regions in the striatum have different roles, they ultimately coordinate and act together at different stages of decision making.

The prefrontal cortex shows activation throughout decision-making (Sarah Rudorf and Todd Hare, Department of Economics, University of Zurich, 2014), namely, the prefrontal cortex shows not only enhanced activity during decisions requiring self-control, but throughout all decision-making processes, but identifying specific regions of the prefrontal the cortexes that are most active in the decision-making process.

A specific network in the brain is active when a person has to decide between different choices in different situations. Studies show the importance of interaction between neurons in two different brain regions within the prefrontal cortex.

The decisions we make affect our daily lives, the way we deliberately commit ourselves is a complex process that we only partially understand. Neuroscientists have identified several brain areas that contribute to and resolve dilemmas (Gold and Shadlen, 2007). However, we are still far from being able to grasp the rigid path from decision to action, because even the most modest decisions involve many areas of the brain and cooperation among many cells (Montague, 2008).

Human choices are sensitive to the way opportunities are presented. The "framing effect" is a violation of standard economic accounts of human rationality, although its deep neurobiology is not understood. The framing effect is related to the activity of the amygdala, which plays a crucial role in the emotional system in mediating decision bias. In individuals, the orbital and medial prefrontal cortex activity predicted reduced sensitivity to the framing effect. Research has highlighted the importance of incorporating emotional processes into models of human choice, and suggests that the brain can modulate the effect of these biased influences on approximate rationality.

\section{Decision Making and Rationality From the Economic and Quantum Aspect}

In contrast to the "rational" economic man, who selfishly makes decisions to optimize his personal usefulness, we have a quantum economic person, who selflessly tangles with other quantum economic people. Happiness is not an individual pursuit that economists can calculate and optimize. Economics is seen as a machine devoid of things like willpower and personal responsibility - Milton Friedman, for example, wrote in 1953 that economics was, in principle, independent of any particular ethical position or normative judgment. Specifically, it may or may be "objective" science, in exactly the same sense as any physical science - quantum economics (if we may call it that) experienced as a living system in which ethics plays an important role. Comparing and remembering the lessons of the crisis where economists were involved in the financial system and were responsible for regulating (for example) through high-paying consultation groups; as in 
quantum physics, the observer is never detached from the system. While neoclassical economics treats "market failures" such as economic inequality and environmental degradation as deviations or external states, from a quantum perspective they appear to more reflect the conflict inherent in money between numerical price and real value, as reflected in the financially sound debt system that prioritizes growth above all else. The theory is based on the findings of thinkers like English chemist Frederick Soddy (who transitioned to economics after receiving the Nobel Prize in 1921 for his work on basic radiation properties), American environmental economist Herman Daly, and many others.

Investment plays an important role not only in the life of the individual, but also in the development of national economies. People save money with the stated goals of protecting the true value of money and making more money. Defining the term investment comes with the expectation of future benefits (Bodie, Kane, \& Marcus, 1988). An investment decision is an important aspect in the investment process that involves choosing one or more investment opportunities for money. Rational decision-making is the result of a systematic decision-making process, and it seeks to achieve the goal of maximizing expected profit (Robbins and Judge, 2007). Rational decision implies the solution of a problem for the decision maker by developing strategies and following certain logical processes taking into account the nature of the problem, the environment and the time (Lin, 2011). The process of rational decision-making according to Mintzberg, Raisinghani and Theoret (1976) takes place in three stages: problem identification, alternative development and selection.

Investment plays an important role not only in the life of the individual, but also in the development of national economies. People save money with the stated goals of protecting the true value of money and making more money. Defining the term investment comes with the expectation of future benefits (Bodie, Kane, \& Marcus, 1988). An investment decision is an important aspect in the investment process that involves choosing one or more investment opportunities for money. Rational decision-making is the result of a systematic decision-making process, and it seeks to achieve the goal of maximizing expected profit (Robbins and Judge, 2007). Rational decision implies the solution of a problem for the decision maker by developing strategies and following certain logical processes taking into account the nature of the problem, the environment and the time (Lin, 2011). The process of rational decision-making according to Mintzberg, Raisinghani and Theoret (1976) takes place in three stages: problem identification, alternative development and selection.

Decision-making can be divided into three types based on the level of rationality. The first type is pure rationality, which is the most rational type in which optimal decisions will be made by decision makers who support unlimited resources, time and knowledge (Gianakis, 2004). The second type is the incremental decision type, which is a less rational model and the judgments are made by evaluating the number of currently available alternatives. Limited rationality is a finite type that is a combination of pure and incremental decision-making that indicates the achievement of certain goals subject to subjective constraints (Simon, 1982, 1991). In general, economic rationality refers to unlimited rationality. This means that the goals are known and well defined, all information is available to them, decision makers are used impartially and choices are consistent.

One of the fundamental aspects of defining investors is never questioned rational or irrational. Peter (1999) defines "irrationality" as evidence of repeated patterns of inconsistency and inability to evaluate market information in the way people make decisions and choices when faced with uncertainty, and should this be called "irrationality" ? The key question is how to judge investor rationality considering inconsistencies and non-competencies and inability to process information? Edwin J. Elton et al. (2004) indicate that in most cases the best indicator is a stock index that "shows" inconsistent and irrational movements in the financial market. When the market moves inconsistently deprived of any economic reasoning, it indicates that investors are investing or disinvesting inconsistently and incompetently. However, it is rational to assume that investors are normal human beings who have mind and heart, have greed, frustration, anger.

\section{Behavior and Financial Decision Making From the Aspect of Newton Law}

Newton's three laws of motion are classical laws of mechanics (1687, Philosophiae naturalis principia mathematica) So, can we use Newton's laws in the twenty-first century in terms of behavioral analysis? There is no equivalent to Newton's laws of motion in the behavioral sciences, and unfortunately Newton's work in mathematics and physics does not provide much information about human behavior. There is no direct translation of " $\mathrm{F}=\mathrm{ma}$ " to the methods of our human madness. Fortunately, there are some general tendencies that have supported much of our behavior.

Behavior is not something that lives in a vacuum. It's intertwined with all intentions, beliefs, knowledge, motivation, personality, human history - and their environment - including everything from the architecture of choice, the grocery store to the light, smell and friends or enemies that surround them. It is necessary to emphasize the interactions of man and their environment, which leads to the exposure of certain behavior at a particular time and place.

Perhaps through the three behavioral laws, we can even now, in the 21 st century, move towards an understanding of human decision-making / financial behavior.

The first law of behavior tends to the status quo, the second law $\mathrm{B}=f(\mathrm{P}, \mathrm{E})$ and the third law of intentional and 
unintentional consequences.

Like the physical properties of the universe, human behavior is extremely complex, as are Newton's laws that describe the movement of physical objects. The intention of the people is to adhere to the status quo if the forces of friction or fuel do not push us out of our way; behavior is a function of the person and their environment; every decision involves compromises and the potential for unintended consequences. With these three laws in mind, we should be able to design better products for people to help them behave better - not just in university labs but also in the private sector, from your savings account to your fitness app.

Newton's first law is essentially a repetition of Galileo's theory of inertia, formulated 50 or more years earlier. Newton's three laws indicate that he was able to distill such incredible volume and complexity of advances in the physical sciences. Its three simple laws formed the basis of innumerable laboratory and field experiments and precursors to Einstein's theory of relativity, but were also used to inform the inventions and innovations of the Industrial Revolution.

However, it should be emphasized that Newton's work in mathematics and physics does not provide much information about human behavior. There is no direct translation of "F = ma" to the methods of our human "madness."

The first law, humans are beings of least resistance. So much of our behavior is triggered by autopilot that it takes considerable effort to take simple actions beyond our usual routine - even when we have the best intentions of changing our behavior. How many times have we decided to bike and how many times have we really done it. We share a bias that describes why we are so bad at this, and it's also called the status quo. The status quo is a powerful force in human behavior, directly analogous to the inertia described by Newton's first law of motion: force is required for change in motion to occur.

The second law of behavior is a function of the person and their environment, $\mathrm{B}=f(\mathrm{P}, \mathrm{E})$. Human behavior is reduced to two essential elements (Kurt Lewin, 1936- its universal equation is still relevant today): the individual characteristics or condition of the person and the environment in which they are located. The Lewin equation does not mean that behavior is a function of a person, or $\mathrm{B}=f(\mathrm{P})$ and - separately - that behavior is also an environmental function, $\mathrm{B}=f(\mathrm{E})$. Of course, there is a lot of research that separately examines person and environment. $\mathrm{B}=f(\mathrm{P}, \mathrm{E})$ contributes to the interaction between the elements. Specifically, we cannot fully understand (or predict) the behavior of X if we only understand $\mathrm{X}$ - who $\mathrm{X}$ is or how he feels or what $\mathrm{X}$ thinks - or if we only understand the environment in which he is. We need both.

There are consequences and potential unintended consequences for every decision. Notwithstanding our attention to the consequences inherent in any decision, losses are often suffered in one area when profits are made in another. This concept of "what do I give up if I make X?" it is known as opportunity cost and it is a type of consequence that is often overlooked. One way to measure such consequences is to classify the potential advantages and disadvantages of the decision and then weigh them (method - signal detection theory). In complex situations, we include some uncertainty using this method to consider the consequences of a particular decision. Because our time and resources are limited, we have to choose how to spend them wisely.

Unintended consequences, when making a decision, we cannot predict future effects that negate or impair the positive aspects of that decision. A classic example of this is the "squeeze" or overactivity effect, where positive behavior (such as exercise) is initially enhanced by an external incentive (financial reward), but the positive effect disappears (and can be reduced to a lower level than before the stimulus is introduced)!) as soon as the incentive is lifted. Rewards like this can increase behavior in the short term but weaken motivation in the long run.

\section{Role of Prediction and Financial Decision Making Quantitely Likely and Decision-Making Processes}

To quote Niles Bohr (Danish physicist, 1922 Nobel Prize winner), "prediction is very difficult, especially in the future," although prediction related the impact of quantum physics on the world in the future, however, it laid the foundation for the difficulties to which theoretical and empirical sciences consistently rely on variable complexity models to make meaningful predictions about future events. Our modern world, thirsty for information and results, often places greater value on the popular belief of future predictions than the intrinsic knowledge gained from adverse events that causes collective herd behavior and cognitive delusions.

Making financial decisions is part of a complex ecosystem that combines behavioral psychology and investment management knowledge. Should decisions be made according to the process in which they were performed or simply their result? Although the answer relies on the definition of the quality of the decision, it largely depends on the compatibility between the decision maker and the assessor (who conducts the second order assessment).

In finance, the decision maker continually faces various (known and unknown) risks that could drastically and quickly affect the value of the portfolio within the portfolio. The process of assessing a portfolio's exposure to risk under normal or black swan conditions cannot (and should not) be adequately covered by a single approach to risk and its variations (let alone a risk-free approach, as is often observed in the field). This indicates the application of a systematic decision- 
making process with the use of a range of risk methodologies to record adherence or deviation of the portfolio probability distribution from normality. The risk management framework requires the execution of scenario simulations. Shocks can cause various macroeconomic or idiosyncratic events, which can therefore be broadly extended to previously unchanged asset choices (systemic or non-diversified risk). Events in recent history are examples of the causes and consequences of crises and vulnerabilities in the financial system, as well as the current global health crisis caused by the COVID 16 pandemic but also by climate change.

Zheng Joyce Wang and other scientists strive to mathematically model our decision-making processes, equations, and axioms that most closely align with human behavior may be those rooted in quantum physics.

"We have accumulated so many paradoxical findings in the field of cognition, and especially in decision-making" (Wang, associate professor of communication and director of the laboratory of communication and psychophysiology at Ohio State University)

"Whenever something that is inconsistent with classical theories, we often refer to it as" irrational. "But from the perspective of quantum cognition, some findings are no longer irrational. They are consistent with quantum theory - and with the way people behave.

When researchers attempt to study human behavior using only classical mathematical models of rationality, some aspects of human behavior do not count. From a classical standpoint, such behaviors seem irrational (Wang).

"In the social and behavioral sciences as a whole, we use probability models a lot" (Wang). "For example, we ask, what is the likelihood that a person will act in a certain way or make a certain decision? Traditionally, all of these models are based on the classical theory of probability - which originated from the classical physics of Newtonian systems. So, social scientists supplement their new knowledge and thinking about quantum systems and their mathematical principles.

Quantum physics deals with ambiguities in the physical world. The state of a particular particle, the energy it contains, its location - everything is uncertain and must be calculated in probability.

Quantum cognition is what happens when people have to deal with ambiguities mentally. We are not always sure how we feel or unclear about which option to choose, or whether we need to make decisions based on limited information.

"...... the mathematical formalism provided by quantum theory is consistent with what we intuitively feel as psychologists. Quantum theory may not be at all intuitive when used to describe particle behavior, but it is actually quite intuitive when used to describe our usually insecure and ambiguous minds. "(Wang). Accordingly in the example

"Schrödinger Cats - A thought experiment in which a cat inside a frame has some likelihood of being alive or dead. Both possibilities have potential in our mind. In this sense, the cat has the potential to become dead or alive at the same time. The effect is called quantum superposition. When we open the box. both possibilities no longer overlap and the cat must be either alive or dead "quantum theory is intuitive in describing our ambiguous and uncertain mind. So, with quantum cognition, it is as if every decision we make is our unique Schrödinger cat.

\section{Conclusion}

The human brain is extremely complex, making it difficult to mimic the processes that occur when, for example, we make a decision. Creating models with the help of quantum information rules and the non-classical logic of the way a person makes decisions is a great challenge. This raises the question of how neurons can generate quantum statistics. There is a lot of such data in experimental psychology, game theory and decision-making, and it is used to describe irrational behavior, among other things.

In physics, it was recognized at the beginning of the twentieth century that some experiments could not be explained by conventional classical mechanics, but the same could be explained by newly discovered quantum theory. This resulted in a new mechanics called quantum mechanics that revolutionized scientific and technological development. It is now recognized that we need quantum mechanics in psychology as well as in economics and finance.

The brain is a very complicated system and we need a coordinated effort that includes almost all branches of the natural sciences: biology, neuroscience, biochemistry, physics, information theory / informatics, medicine, pharmacology, but also the social sciences. There has always been an interface between psychology and physics (psychophysics). A unique scheme of brain and mind dynamics, in accordance with all known laws of physics, including energy saving. Thus, our consciousness is nothing but a localized aspect, a global, integrative process and a path to Homo Quantum.

In the early twentieth century, physics showed that some experiments could not be explained by classical classical mechanics, but the same could be explained by quantum theory. This resulted in a new mechanic, namely quantum mechanics, which revolutionized scientific and technological development. In the early twenty-first century, it was recognized that some experiments related to human decision-making processes and could not be explained by conventional classical decision theory, but models based on quantum mechanics can explain the same model. It is also 
the path of the need for quantum mechanics in psychology as well as in economics and finance. In this paper, we try to improve and explain the current understanding of the applicability of quantum mechanics to the human decision-making process. We use postulates analogous to the postulates of quantum mechanics. The interaction of financial decisionmaking, quantum physics and the human brain indicates the intricate complexity of financial decision-making towards the application of new knowledge (quantum financing, quantum management) to meet the challenges of financial market vulnerabilities in times of pandemic and climate change,

It is important to monitor and work on, learn from new research and algorithms to reduce unknowns and to help us understand how the human brain learns to adapt to the complexities and uncertainties of learning and decision making. The computer and neural mechanism for learning the human amplification goal opens up the possibility of introducing key elements of human intelligence into artificial intelligence algorithms. Such insights could ultimately use us for computational models to reverse engineering learning about human reinforcements. It should be borne in mind that the development of new technologies and artificial intelligence will completely change our financial environment, such a process has already begun.

The frameworks for studying the functional relationships between the tools of neuroscience, neurofinance and psychology on the one hand, and quantum physics / quantum mechanics and neurophysiology on the other, are analyzes of the benefits of using quantum-like modeling models, while there are limitations such as lack of experimental experiments. (high costs of interdisciplinary research).

\section{Reference}

Aerts, D. (2009). Quantum structure in cognition. Journal of Mathematical Psychology, 53, 314-348. https://doi.org/10.1016/j.jmp.2009.04.005

Aerts, D. (2014). Quantum theory and human perception of the macro-world. Front. Psychol, 5, 554. https://doi.org/10.3389/fpsyg.2014.00554

Aerts, D. et al. (2013). Concepts and their dynamics: A quantum theoretic modelling of human thought. Topics in Cognitive science, 5, 737-772. https://doi.org/10.1111/tops.12042

Aerts, D., Broekaert, J., Gabora, L., \& Sozzo, S. (2016a). Generalizing prototype theory: a formal quantum framework. Front. Psychol, 7, 418. https://doi.org/10.3389/fpsyg.2016.00418

Aerts, D., Broekaert, J., Gabora, L., \& Sozzo, S. (2016b). Quantum Structures in Cognitive and Social Science, 7. Lausanne: Frontiers Media SA. https://doi.org/10.3389/fpsyg.2016.00577

Allais, M. (1953). Econometrica, 21, 503. https://doi.org/10.2307/1907921

Basieva, I., \& Khrennikov, A. ( 2017 ). Decision-making and cognition modelling from the theory of mental instruments. The Palgrave Handbook of Quantum Models in Social Science. Applications and Grand Challenges. Haven E and Khrennikov A (eds). Palgrave Macmillan UK, 75-93. https://doi.org/10.1057/978-1-137-49276-0_5

Busemeyer, J. R., \& Bruza, P. D. (2012). Quantum Models of Cognition and Decision. Cambridge: Cambridge University Press

Busemeyer, J. R., \& Wang, Z. (2015). What is quantum cognition, and how is it applied to psychology? Curr. Direct. Psychol. Sci, 24, 163-169. https://doi.org/10.1177/0963721414568663

Campbell, N. R. (1957). Foundations of Science: Philosophy of Theory and Experiment. New York: Dover.

Edwards, W., \& Exp, J. (1955). Psychol, 50, 201. https://doi.org/10.1037/h0041692

Ellsberg, D. (1961). Risk, ambiguity, and the Savage axioms. The quarterly journal of economics, 75. https://doi.org/10.2307/1884324

Elton, E. J., Gruber, M. J., \& Busse, J. A. (2004). Are investors rational? Choices among index funds. The Journal of Finance, 59(1), 261-288. https://doi.org/10.1111/j.1540-6261.2004.00633.x

Gabora, L., \& Aerts, D. (2005). Evolution as context-driven actualisation of potential: toward an interdisciplinary theory of change of state. Interdisc. Sci. Rev, 30, 69-88. https://doi.org/10.1179/030801805X25873

Haven, E., \& Khrennikov, A. (2013). Quantum Social Science. New York, NY: Cambridge University Press. https://doi.org/10.1017/CBO9781139003261

Haven, E., \& Khrennikov, A. (2017). The Palgrave Handbook of Quantum Models in Social Science. London: Macmillan Publishers Ltd.,. https://doi.org/10.1057/978-1-137-49276-0

Helmholtz, H. von. (1930). Counting and measuring. Translated by C. L. Bryan; uzv. and notes by H. T. Davies. New York: D. Van Nostrand. 
Von Neumann, J., \& Morgenstern, O. (1953). Theory of Games and Economic Behavior, Princeton University, Princeton.

Khrennikov, A. (2017). Social laser: action amplification by stimulated emission of social energy. Philosophical Transactions A. https://doi.org/10.1098/rsta.2015.0094

Khrennikov, A., \& Haven, E. (2009). Quantum mechanics and violations of the sure-thing principle: The use of probability interference and other concepts. Journal of Mathematical Psychology, 53, 378-388. https://doi.org/10.1016/j.jmp.2009.01.007

Khrennikov, A., \& Emmanuel, H. (2013). Quantum Social Science. CUP, 1st Edition.

Khrennikov, P., \& Patra, S. (2019), Asset Trading under non-classical uncertainty. Physica A , 521, 562-577. https://doi.org/10.1016/j.physa.2019.01.067

Knight, F. H. ( 1921). Risk, Uncertainty, and Profit, New York.

Oliver, A. (2018). Your money and your life: risk attitudes over gains and losses. J. Risk Uncertain. 57, 29-50. https://doi.org/10.1007/s11166-018-9284-4

Penrose, R. (1989). The Emperor's new mind. Oxford University Press.

Peres, A. (2002). Quantum Theory: Concepts and Methods. New York, NY: Kluwer Academic Publishers. https://doi.org/10.1007/0-306-47120-5

Peter, L. B. (1999). Against the God. Accessed from www.investorhome.com

Savage, L. J. (1954). Foundations of Statistics. Wile, New York.

Susskind, L., \& Art, F. (2014). Quantum Mechanics: The theoretical minimum, Basic Books.

Tversky, A., \& Kahneman, D. (1992). Advances in prospect theory: Cumulative representation of uncertainty. Journal of Risk and uncertainty, 263-291. https://doi.org/10.1007/BF00122574

\section{Copyrights}

Copyright for this article is retained by the author(s), with first publication rights granted to the journal.

This is an open-access article distributed under the terms and conditions of the Creative Commons Attribution license which permits unrestricted use, distribution, and reproduction in any medium, provided the original work is properly cited. 